\title{
Study on the Common Misunderstandings and Corrective Methods of Piano Teaching and Performance
}

\author{
Linhan Yu \\ Wenshan University, Wenshan, Yunnan, 663099
}

Keywords: Piano teaching and performance; common misunderstandings; corrective methods; analysis

\begin{abstract}
Piano teaching and performance work is very professional and technical. Therefore, it is very important for teachers to teach students the correct learning methods and guide students to master relevant skills when carrying out piano teaching. At this stage, many students are blind and random in learning piano. They do not master scientific and standardized playing methods, resulting in many learning misunderstandings, wasting a lot of time and energy, and the quality of learning is difficult to improve. This paper focuses on common misunderstandings and corrections in piano teaching and performance.
\end{abstract}

\section{Introduction}

Piano performance is a subjective and rational thing. An excellent pianist must have a unique and profound understanding of the tracks, perfect skills, scientific logic, strong desire to perform, and dedicated stage performances. Therefore, in the process of teaching, the piano teacher should discover the problems in the students' performance in time, cultivate the students' passion to play the piano and express the music, guide the students to improve their understanding and technical ability, correct their own deficiencies, and become an excellent piano. Talent [1]. Next, talk about common misunderstandings and corrections in piano teaching and performance.

\section{The reading spectrum misunderstanding and correction method}

In the process of piano performance, the player only fully and accurately expresses the information on the score, which is the basis and premise for accomplishing his mission. Students who have just touched the piano are unfamiliar with the staff and the piano keyboard, and need to look at the keyboard practice. If you don't help the students to correct this misunderstanding in time, in the long run, the students can't play the piano while watching the spectrum, and they can't effectively improve the reading ability.

At this stage, the key factor affecting the quality of the piano performers is the psychological factors that the players are eager to achieve. In fact, the process of reading the spectrum is a process of savoring. Different notes are reflected on the fingers through a combination of different ways. The number of sounds in each measure and each beat is different. Therefore, when reading the spectrum, you should determine the relatively slow playing speed according to the paragraph with more notes and complex rhythm, and always keep this speed to control the whole song. In the scope of his own ability, a complete initial impression of the whole piece of music is formed, thus laying a solid foundation for further practice. If the player has a eagerness to seek success, in the process of playing, his heart is full of anger, it is easy to make mistakes. As a performer, if you practice in the wrong way of reading at the beginning, it will be difficult to correct in future exercises.

Based on this, if a pianist wants to develop a correct and good reading habit, he must be steady and steady. First of all, in the early stage of reading the reading spectrum, focus on cultivating reading, reading, and backing various symbols in advance, so that the eyes can walk in front of the hands; when practicing new tracks, first check the pitch, time value, fingering, playing method, strength and other factors. , forming a complete first impression of the track. Secondly, it is necessary to strengthen the understanding of the piano music, in-depth study of the creative 
background of the performance of the music, the thoughts and feelings expressed by the author, the content of the song and other factors. In addition, during practice, you should always keep reading in the correct posture, avoiding excessive focus on a certain part, limiting the visual field, and focusing on improving the integrity and fluency of the reading spectrum [2].

\section{The misunderstanding and correction method}

The staccato we often say is the "jumping" method, which is more common in relaxed and happy music. When a piano beginner usually breaks, he mistakenly pulls his wrist and elbow up, causing the unique brilliance and elasticity of "jumping" to disappear instantly. Secondly, the continuous play of "jumping" requires the trainer to maintain muscle tension at all times, eventually leading to a sore arm.

Based on this, in the teaching process, the teacher should help the students to accurately grasp the "jumping" action essentials: the touch key is fast and the time is short, the key is quick; the fingertip is firm and flexible; the finger touches the key surface and so on. In order to reduce the difficulty of students' understanding, teachers can create relevant teaching situations: ask students to close their eyes, breathe at a constant speed, fantasize that their hands become a ball, imagine the keys as elastic trampolines on the playground, and their fingers are jumping The children rebounded very high without jumping once, letting the students find the feeling that the fingers relaxed and jumped, and each jump was bounced back by the keys. Therefore, when the staccato is played, the fingertips must be hard and fast to touch, so that the reaction force of the quick touch key can be fully utilized, and the natural drop and the homeopathic bounce can be played to play the rich sound.

\section{The shortcomings and corrective methods of fast and slow training}

Many piano students ignore the slow training while playing a fast technical paragraph of a song, and directly and repeatedly make quick contact, which leads to the ability to play fast passages with the piano, and the performance is worse. The fundamental reason is that students neglect the importance of slow training. They do not correctly realize that a single quick practice can not only improve the speed, but also cause the movement of the playing to be distorted. In fact, the steady and steady training is the basis and premise of fast practice. As long as you keep the fast moving action essentials during slow training, each action should still be fast and fast like fast play, so that it looks like speed. Very slow, but it really masters the relationship between sound and rhythm, resulting in better performance [3].

On the other hand, it is important to strengthen the slow training from the perspective of skill practice to consolidate the effect of fast play. Because the normativeness and accuracy of technical movements need to be dominated and corrected by the subjective consciousness of the performer, and this can only be achieved at a relatively slow speed. If the player initially ignores the conscious normative requirements, then once the action is formed Habits, and then want to correct, it is very difficult. It is worth mentioning that when we play a very perfect piece of practice, we must also have a sense of speed and slowness, because the habitual movements in the piano performance will gradually become blurred and inaccurate in the performance. As a result, it is easy to make mistakes in the performance. Secondly, the unconscious and inattentive piano performance training will slowly lose the vividness of the musical performance, making the delicate and delicate performance of the performance become unseen, making the artistic performance of the piano performance unappealing. Inconclusive. It can be seen that when learning piano, you must pay attention to fast and slow practice.

\section{Misunderstandings and corrective methods in understanding musical expression techniques and understanding of musical terms}

The terminology we often refer to refers to the key tips that piano composers provide to 
performers to understand and express music. If the player does not correctly understand the signs, symbols, and musical terminology in the score, then the performer becomes passive, blind, and unable to start when expressing the music. It can be seen that the precise understanding and interpretation of the musical terms and expression terms in the piano works by the performers is the key to perfectly expressing the composer's thoughts and emotions. For example, "Crese" means "gradually strong”, that is, gradually becoming stronger, “dimin” means "fading out” and gradually weakening. These two are the most common musical terms in piano works, but they are also in the process of student performance. The most common problem-prone term, most students immediately increase their strength when they see "Cress". When they see "dimin", they immediately let go. They don't know that "gradual strength" means "gradually stronger from weak". "Fade out" refers to "gradual weakening from strength", which is a gradual process [4].

Based on this, in the teaching process, the teacher must warn the students to see the "Cress", and then slowly increase from the weak bounce, when you see "dimin", you must gradually weaken from the strong bounce, so that After the students have established the correct concept and strengthened the practice, they will no longer regard "Cress" and "dimin" as a strong and weak contrast. For another example, "accel" means gradually accelerating. It is based on the speed of the previous paragraph. The speed is gradually accelerated. Many people will misunderstand it as faster. "Piumosso" means speeding up, referring to the speed of playing in the previous paragraph. On the basis of the sudden speed up playing. However, in the actual process, it is easy for the player to confuse "accel" with "piumsso", so that mistakes occur during the piano performance. Therefore, the piano teacher must attract the attention of the students in the teaching process, correctly distinguish the differences between the two words, avoid mistakes, and thus present a more perfect piano performance.

\section{Misunderstandings and corrective methods of playing posture}

Most of the students in the beginning of the piano, in order to meet the requirements of the opponent pointed out by the teacher, that is, we often say that the palms and fingers are half-bent, as if the palm of the hand holds the egg and the palm and fingers are very nervous, so As a result, the weight of the arm is suspended high, making the shoulders, arms, elbows, and wrists stiff and unsmooth, so that the force cannot be transmitted to the fingertips, and the muscles of the palms become tense, making the fingers unable to Independent and flexible use. The reason is that students do not really understand the requirements of the "correct" hand type. It can be seen that as a piano teacher, students must be guided to correctly realize that using the "correct" hand is not the purpose, but the result of relaxation, is the most direct and effective way to detect whether the palm and the arm are relaxed during the performance. . Only when the player is completely relaxed can the palm, arm and fingers show the perfect, natural semi-bend state. Only when the students fully understand and grasp the relationship between the hand shape and the relaxation can they feel the natural sagging of the arm and the semi-bending state of the hand into a relaxed state in the staccato [5]. On this basis, the teacher is strengthening the student's connection to the legato, training the finger to bend and move freely on the basis of standing. When walking stops, feel the arm naturally sag and the hand is curved. After a long period of training, It is easy for students to master this correct playing posture so that they can understand that the correct hand is a relaxed state and is the result of relaxation.

All the piano teachers have always asked students to raise their fingers during the teaching process. Many students struggled to lift their fingers high during the performance. However, after a period of practice, the students not only did not improve. The strength and speed of playing, the fingers become inflexible, and even some students have stiff arms, wrists, and palms. The reason is that students do not really grasp the meaning, purpose and application skills of using "high finger", which leads to misunderstanding.

Based on this, the piano teacher must guide the students to master the use of "high finger" in order to exercise the independence and flexibility of the finger and strengthen the power of the finger. The "high finger" is often applied to piano beginners and lack of fingers. Flexibility, 
independence, and not all performances require the use of "high finger". First of all, the performance technique of "high finger" is usually to use the movement of the interosseous muscle to raise the finger. It is forbidden to pull the finger that has already been dropped, but to use the deep flexor of the finger to truly improve the finger. Independence and flexibility. It is worth mentioning that in the process of raising the finger, the player must lift the finger to be played separately, and the adjacent finger is in a relaxed state. In this way, the repeated use of the irrelevant finger can be effectively avoided, resulting in the finger. Tension, fatigue, and even cause sports injuries [6]. Secondly, during the performance, if you need to use the "high finger" to play, the player's finger will be lifted and the speed of the key will be very fast. The moment the finger touches the key should not be stopped, but should be directly played. In this way, the sound of the piano is full and powerful, and the purpose of raising the finger. Never break a coherent playing action into several actions to complete separately. For example, some students use the "high finger", the finger lifts very fast, but the touch speed is very slow; another example, some students quickly lift their fingers and quickly fall back to the keys, but pause Just press the key. These erroneous "high finger" play will greatly reduce the fullness, smoothness and power of the piano playing sound, not only making the action advantage of "high finger" disappear, but also leading to finger independence and flexibility training. Therefore, teachers and students must attach great importance to this during the teaching process.

For a long time, in the process of piano teaching and performance, the misunderstanding of shoulders is often on the shoulder. The shoulders mentioned here refer to the player's shoulders rising because of excessive tension and excessive force on the shoulders. Once the player is in a state of rising shoulders, the strength of his arm will not go down, and the shoulder joints will not be able to flexibly move with the arms and elbows, thus greatly reducing the performance.

Based on this, the piano teacher should guide and assist the students to do related exercises before practicing the piano and playing, thus relaxing the muscles and adjusting the emotions. For example, to bend the waist, let the students stretch their arms as much as possible, then put their arms on the chest, do the back and forth cross movements, then move the shoulder joints, turn the head and neck, and move the wrists to relax their arms and adjust their mood. .

\section{Conclusion}

In summary, in the process of carrying out piano teaching activities, teachers must first guide students to establish correct learning concepts, playing concepts, and master a variety of scientific and correct playing skills. Secondly, under the guidance of these correct ideas and methods, we will continue to strengthen training, find out the misunderstandings in piano performance in time, and take effective measures to solve them. Gradually cultivate correct and good playing habits and comprehensively master the piano performance. Law, skills, and ultimately become an excellent pianist.

\section{References}

[1] Gen Lei. On the Cultivation of Music Sense in Piano Teaching — The Way and Method of Music Sense Training[J]. Contemporary Music, 2016(8).

[2] Sun Wei. On the problem of touch-key skills in piano performance and teaching[J]. Northern Music, 2016, 36(7).

[3] Wu Chao. Research on the Cultivation of Stage Performance Consciousness in Piano Teaching_-The Students' Active Thinking on the Piano Performance Problem and the Answer to the Spontaneous Question[J]. Northern Music, 2017, 37(5).

[4] and Gui Lian. On the Importance of Playing Hands in Piano Teaching [J]. Popular Literature, 2016 (11).

[5] Zhu Wei. Mistakes and Correction Strategies of Students in Piano Teaching[J]. Journal of 
Jiamusi Vocational College, 2016(05).

[6] Yi Xiaohong, Liu Peifa. Analysis of the Importance and Method of Piano Performance Speed[J]. Big Platform,2010(01). 